\title{
Analyse dimensionnelle en interaction fluide-structure
}

\author{
Dimensional analysis in fluid-structure interaction
}

\author{
par E. de Langre \\ LadHyX, Ecole Polytechnique, Palaiseau
}

Mechanical interactions between fluids and solids give rise to a large variety of physical phenomena. This paper illustrates this diversity and points out the looseness of the terminology of today. A more systematic analysis which is based on dimensional analysis is then proposed. The most usual dimensionless numbers are built and these are used for classifying examples.

\section{I 口INTRODUCTION}

\subsection{Quelles interactions?}

Le titre du colloque dans lequel s'inscrivait cette contribution est «Interactions Mécaniques entre Fluides et Structures ". Nous ne considérerons donc pas ici les interactions pourtant réelles faisant intervenir la thermique à l'interface ni les influences chimiques ou même biologiques entre ces milieux. Ce sont des sujets passionnants, parfois liés à la Mécanique.

En se restreignant donc au domaine de la Mécanique nous proposons également d'exclure ici les configurations où le fluide et le solide occupent à l'échelle macroscopique le même espace. C'est le cas des milieux poreux ou des suspensions dont la modélisation fait appel à des concepts assez différents.

Enfin, nous supposerons qu'il n'y a pas d'échange de masse à l'interface tel que dissolution, solidification ou érosion.

Quand ces interactions existent-elles ? Pour nous, ce seront les situations dont la modélisation nécessite l'utilisation conjointe de la Mécanique des solides et de la Mécanique des fluides c'est-à-dire la prise en compte, a priori nécessaire, des mouvements des deux milieux. Comme on le verra plus loin, des hypothèses simplificatrices permettent dans certains cas d'alléger cette description simultanée des deux mouvements.

\subsection{Exemples}

Dans le cadre que nous nous sommes fixé, il reste encore une très grande variété d'interactions possibles, comme les différentes contributions de ce colloque l'illustreront. Citons ici quelques cas pour fixer les idées.

Cas A. Le 14 septembre 1997, un chasseur F117 s'est écrasé lors d'un meeting aérien, suite à des «vibrations excessives de flottement d'un aileron " [1].

Cas B. Il existe actuellement dans le monde plus de 2000 barrages souples, formés de longs tubes de plusieurs mètres de diamètres et plusieurs dizaines de mètres de long, gonflés à l'eau ou à l'air. Leur comportement mécanique pendant les phases de gonflage, de remplissage du barrage ou de séismes fait l'objet de nombreuses études [2].

Cas C. Certains dispositifs de lecture-écriture sur disque informatique fonctionnent avec une tête planant à moins de $1 \mu \mathrm{m}$ au-dessus de la surface du disque, grâce à l'air entraîné par ce dernier. La stabilité de cette tête a une grande importance pour la fiabilité de l'appareil [3].

Cas D. La très faible traînée du dauphin a entrainé depuis les années 60 de nombreuses recherches sur le lien entre la viscoélasticité d'une paroi et l'apparition de la turbulence dans la couche limite [4].

Cas E. L'effet dit "Castafiore", dans lequel une cantatrice brise un verre par une note aiguë a été immortalisé par Hergé, et repris dans une publicité pour un téléphone. Cet effet est-il un mythe ?

Cas F. On sait aujourd'hui construire des systèmes électro-mécaniques (moteurs, actionneurs, capteurs) dont les tailles caractéristiques sont de l'ordre de $100 \mu \mathrm{m}$ (« MEMS », ou Micro-Electro-Mechanical Systems). L'effet de la viscosité de l'air sur leur mouvement est importante et en perturbe le fonctionnement [5].

Dans ces quelques exemples, l'utilisation conjointe de la Mécanique des solides et de la Mécanique des fluides semble nécessaire. Pourtant l'intuition suggère que les mécanismes physiques en jeu dans ces interactions sont très diffé- 
rents. Avant toute analyse détaillée, leur variété suggère de se munir d'un outil de classification.

\section{- 1.3 Terminologie}

La terminologie courante dans le domaine qui nous intéresse ici n'aide pas toujours l'ingénieur ou le chercheur à se faire une représentation claire des différents phénomènes physiques et de leur importance relative dans chaque cas.

En consultant quelques livres, cours, ou articles récents on trouve en français les termes suivants : interaction fluidestructure, couplage fluide-structure (faible ou fort), couplage fluide-élastique, vibro-acoustique, acousto-mécanique, aéroélasticité (dynamique, statique, quasistatique, pseudo-statique,...), hydro-élasticité, flottement, galop, vibrations induites par les écoulements, vibrations induites par vortex (VIV), accrochage, etc. La terminologie anglaise propose fluid-structure interaction, flow-induced vibration (FIV), vibroacoustics, aero-hydroelasticity, galloping, flutter, buffeting, flapping,...

Ces termes ne désignent pas toujours des réalités identiques d'un auteur à l'autre. Par exemple, l'expression générale "Interaction Fluide-structure " désigne parfois uniquement les interactions avec les fluides sans écoulement permanent. Nous n'essayerons pas de donner une définition unanimement acceptée de ces termes, dont l'usage varie. Nous proposons dans le paragraphe suivant une démarche d'analyse dimensionnelle qui doit permettre de construire une classification non ambiguë des différentes situations.

\section{II - ANALYSE DIMENSIONNELLE}

\subsection{Quelques principes}

L'utilisation de l'analyse dimensionnelle est bien connue des expérimentateurs qui veulent respecter des similitudes. Elle a également une richesse propre car elle donne des outils, les nombres adimensionnels, qui permettent de quantifier l'importance relative des effets physiques et donc de classer les problèmes par familles de phénomènes. L'exemple le plus classique en mécanique des fluides est celui du nombre Reynolds, qui donne un ordre de grandeur du temps de diffusion visqueuse par rapport au temps de convection. On peut classer, pour une géométrie donnée, la structure des écoulements à partir de ce nombre de Reynolds.

La démarche de construction de nombres adimensionnels est simple, décrite dans la plupart des cours de mécanique des fluides $[6,7,8,9]$.

Si un système physique est décrit par $N$ grandeurs (dimensions, viscosité,....), cette description peut se réduire à $N-R$ nombres adimensionnels, où $R$ se déduit simplement de l'analyse des dimensions de chaque grandeur (longueur, masse, temps,...). C'est la simple conséquence du fait que les lois physiques sont indépendantes des unités dans lesquelles on les exprime. On ne fait donc appel à aucune équation particulière vérifiée par ces grandeurs, mais le choix de leur liste est déjà un choix de modélisation.

\subsection{Application}

Appliquons cette démarche aux interactions mécaniques entre fluides et structures, en commençant par un cas générique (fig. I).

Dans le fluide on connaît un ordre de grandeur de la vitesse, $U$, de la pression $p$, de la masse volumique $\rho_{F}$, de la viscosité $v$ et de la vitesse du son $c$.

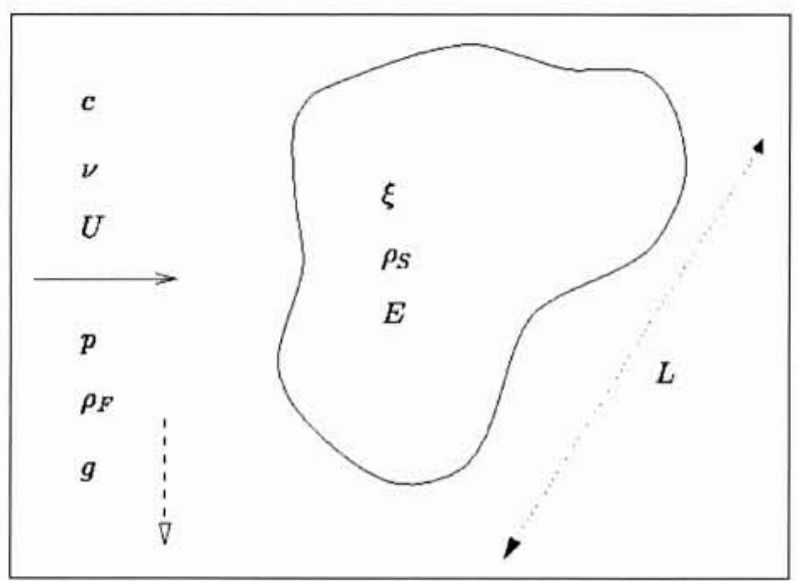

1. Paramètres de description du fluide et du solide.

Dans le solide, on se donne par exemple l'ordre de grandeur des déplacements, $\xi$, la masse volumique $\rho_{S}$ et le module d'Young $E$.

Enfin on se donne un dimension caractéristique du problème, $L$, et la gravité $g$.

A partir des dix grandeurs ci-dessus on peut construire sept nombres adimensionnels, dont le choix n'est pas unique. Prenons par exemple

$$
\begin{gathered}
R_{E}=\frac{U L}{v} ; F_{R}=\frac{U}{\sqrt{L g}} ; M=\frac{U}{c} ; \Pi=\frac{\rho_{F} U^{2}}{p} ; \mathscr{D}=\frac{\xi}{L} ; \\
\mathscr{G}=\frac{\rho_{S} g L}{E} ; \mu=\frac{\rho_{F}}{\rho_{S}} .
\end{gathered}
$$

Les quatre premiers nombres sont bien connus des mécaniciens des fluides. Ce sont les nombres de Reynolds, de Froude, de Mach et de pression dynamique réduite. Les deux suivants traduisent pour le solide la mesure de l'effet de grands déplacements $(\mathscr{D})$ et l'importance des déformations liées à la pesanteur ( $\mathscr{G})$. Le dernier, le nombre de masse $\mathcal{M}$, est le seul qui fasse intervenir simultanément une caractéristique du fluide $\rho_{F}$ et une caractéristique du solide $\rho_{S}$.

A partir de ces nombres, on peut exprimer de façon plus précise les différentes hypothèses que l'on peut faire sur les interactions entre les deux milieux.

Par exemple faire de la mécanique des fluides sans prendre en compte le mouvement du solide se traduirait par un choix restrictif des paramètres adimensionnels qui influent sur le champ de vitesse. La valeur locale $u$ de celui-ci serait alors de la forme :

$$
\frac{u}{U}=F\left[R_{E}, F_{R}, M, \Pi\right]
$$

Au contraire, si l'on admet la possibilité d'interactions, la vitesse du fluide peut être influencée par la mouvement du solide et on a alors

$$
\frac{u}{U}=F\left[R_{E}, F_{R}, M, \Pi ; \mathscr{D}, \mathscr{G} ; M\right] .
$$

De la même façon, le déplacement $\underline{X}$ en un point dépend de la vitesse de l'écoulement par

$$
\frac{X}{\xi}=G\left[R_{E}, F_{R}, M, \Pi ; \mathscr{D}, \mathscr{G} ; M\right] .
$$

Il faut noter que l'on n'a pas précisé quelle était la manière dont ces nombres influent dans les expressions $(2,3)$ ou (4). C'est l'exploration par l'expérience ou par le calcul de ces espaces à quatre ou sept dimensions qui permet de le savoir. 


\subsection{Autres nombres adimensionnels}

Les sept nombres proposés plus haut peuvent être combinés à l'infini pour donner de nouveaux nombres, plus parlants dans tel ou tel problème. Les plus courants, que nous discuterons plus loin, sont

Vitesse réduite

$$
V_{R}=\frac{U}{\sqrt{\frac{E}{\rho_{S}}}}=F_{R} \sqrt{\mathscr{G}},
$$

Nombre de Stokes

$$
S_{T}=\frac{\sqrt{\frac{E}{\rho_{S}}} L}{v}=\frac{R_{E}}{V_{R}},
$$

Nombre de Froude dynamique

$$
F_{D}=\frac{\sqrt{\frac{E}{\rho_{S}}}}{\sqrt{L g}}=\frac{F_{R}}{V_{R}},
$$

Nombre de compressibilité

$$
\begin{aligned}
& \mathscr{C}=\frac{\sqrt{\frac{E}{\rho_{S}}}}{c}=\frac{M}{V_{R}}, \\
& \mathscr{C}_{y}=\frac{\rho_{F} U^{2}}{E}=V_{R}^{2} \cdot M .
\end{aligned}
$$

Il peut également être plus pertinent de choisir d'autres grandeurs pour caractériser le système. Par exemple, la dynamique du solide peut être plus facilement décrite par une fréquence d'oscillation, $f$, que par la vitesse de propagation des ondes élastiques $\sqrt{\frac{E}{\rho_{S}}}$. Les nombres ci-dessus deviendraient alors

$$
V_{R}=\frac{U}{f L} ; S_{T}=\frac{f l^{2}}{v} ; F_{D}=\sqrt{\frac{f^{2} l}{g}} ; \mathscr{C}=\frac{f L}{c} .
$$

Enfin il faut souvent augmenter le nombre de grandeurs physiques pour décrire plus précisément la réalité. Par exemple, si le solide est très allongé, sa dimension transverse, $d$, doit être prise en compte. On introduira alors un nouveau nombre, l'élancement

$$
\varepsilon=\frac{L}{d},
$$

et les nombres définis plus haut s'exprimeront plutôt avec $d$ ou $L$ selon la direction de l'écoulement par rapport au solide et leur signification propre.
De même, si l'écoulement du fluide développe une oscillation propre de fréquence $f_{F}$, par exemple dans le sillage du solide, on définit le nombre de Strouhal

$$
S=\frac{f L}{U} .
$$

\subsection{Signification physique des nombres adimensionnels.}

Les nombres adimensionnels ne sont pas simplement le résultat d'une algèbre sur les unités physiques. Ils expriment le quotient de deux grandeurs dimensionnelles et même plusieurs quotients de grandeurs dimensionnelles. Les interprétations que nous donnons ci-dessous portent sur des échelles de temps caractéristiques de chaque mécanisme de propagation. On peut les reformuler en termes de longueurs, de vitesses ou d'efforts. La figure 2 illustre les mécanismes physiques que nous allons discuter.

La vitesse réduite, $V_{R}$, est le rapport entre le temps caractéristique du mouvement du solide, $1 / f$ et l'ordre de grandeur du temps de passage du fluide le long du solide, $L / U$. Ainsi, pour $V_{R} \ll<1$, le solide ne "voit pas " le fluide bouger pendant la durée caractéristique de son mouvement. Inversement, $V_{R} \gg 1$ signifie que le déplacement du solide est imperceptible pendant le temps que le fluide met à parcourir celui-ci.

De la même façon, le nombre de Stokes, $S_{T}$, exprime le rapport entre l'échelle de temps de diffusion visqueuse, $\mathrm{L}^{2} / v$, et le temps caractéristique du mouvement du solide, $1 / f$. A grand nombre de Stokes, les effets visqueux seront donc négligeables. C'est donc l'analogue du nombre de Reynolds, mais construit sur le temps d'oscillation du solide et non pas sur le temps de convection, comme l'exprime la relation $S_{T}=R_{E} / V_{R}$.

Le nombre de Froude dynamique, $F_{D}$, n'a de sens que quand des ondes de gravité peuvent exister, par exemple avec une surface libre. Il représente alors le rapport entre un temps de propagation des ondes de gravité $L / \sqrt{g L}$ et le temps caractéristique du mouvement du solide. Si $F_{D}$ est grand, cela signifie que la diffusion d'énergie par rayonnement des ondes de surface est lente à l'échelle de temps du solide. On la négligera donc. Cela ne signifie pas qu'il ne faille pas prendre en compte la condition de surface libre, qui peut modifier la masse ajoutée. Inversement, un faible nombre de Froude impose de prendre en compte ce rayonnement, mais celui-ci aura un effet très différent selon que les ondes reviennent ou pas. Par exemple, le mouvement vertical d'un bateau dans un bassin étroit produit des effets distincts d'un mouvement en pleine mer.

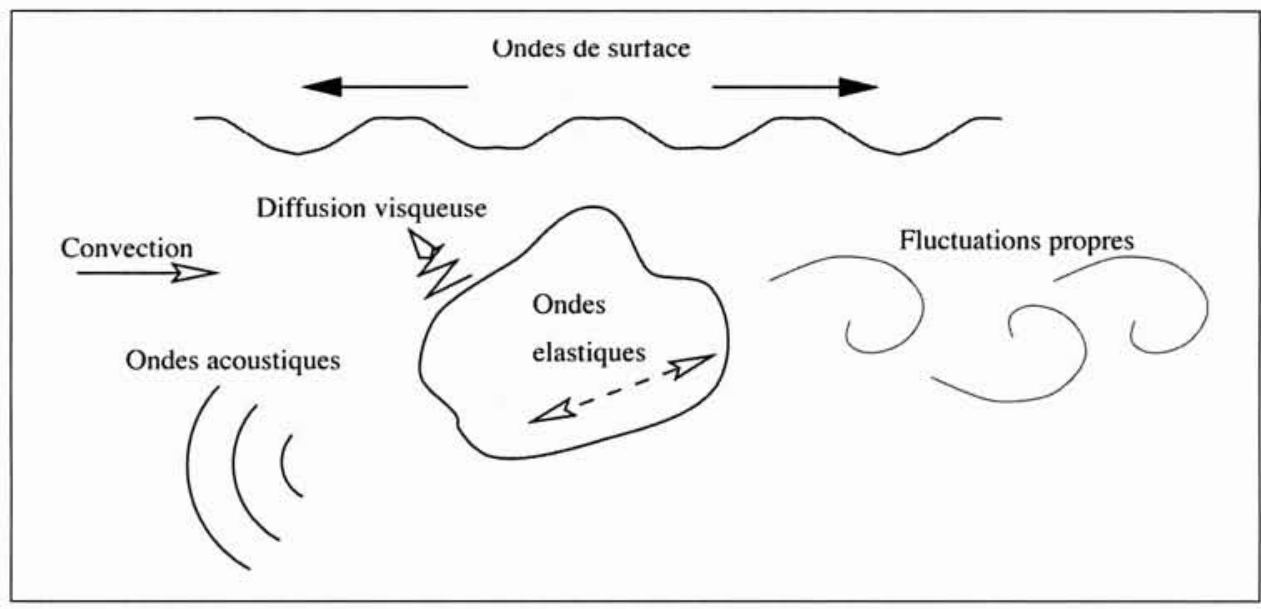

2. Mécanismes physiques à l'origine des différentes échelles de temps. 
De même le nombre de compressibilité $\mathscr{C}$ est le rapport entre un temps de propagation des ondes acoustiques $L / c$ et le temps caractéristique du mouvement du solide. On peut l'interpréter selon le même schéma que le nombre de Froude dynamique. Pour $\mathscr{C} \gg 1$ le rayonnement acoustique est lent et donc la compressibilité du milieu doit être prise en compte localement. Pour $\mathscr{C} \ll 1$ l'information acoustique est, à l'échelle de temps du solide, instantanément transmise dans le fluide. Là encore, selon les conditions aux limites, la situation doit être analysée plus finement. Par exemple, un tube à piston, rempli d'eau, sur lequel on appuie très lentement, donc avec $\mathscr{C}<<1$, fait travailler la compressibilité du fluide, qui doit évidemment être prise en compte. Mais ce n'est pas le cas du mouvement vertical du navire, dans le bassin ou à la mer, à même valeur de $\mathscr{C}$ !

Enfin le nombre de masse $\mu$ est directement un rapport de masse. Il prend tout son sens à basse vitesse réduite, dans les effets de masse ajoutée.

Le nombre de Cauchy $\mathscr{C}_{y}$ est, lui, une mesure des déformations induites par la pression dynamique. Il prend tout son sens à haute vitesse réduite, dans les effets de raideur ajoutée.

Il est clair que $M \ll<$ ou $\mathscr{b}_{y} \ll 1$ impliquent une faible influence du fluide sur le solide, relativement à sa propre masse ou sa propre raideur.

Nous terminons par le nombre de Strouhal. Il est caractéristique du rapport entre deux échelles de temps du fluide $L / U$ et $1 / f_{F}$. Certains auteurs le confondent avec la fréquence réduite $f L / U$, inverse de la vitesse réduite. Cette confusion provient du fait que l'accrochage tourbillonnaire se produit souvent à la résonance $f_{F}=f$.

\section{III — MODÉLISATIONS}

\subsection{Classement par nombres adimensionnels}

En estimant l'ordre de grandeur des nombres adimensionnels pour une situation donnée, on obtient donc une estimation de l'importance relative des différents mécanismes. C'est une indication qui permet de choisir les approximations que l'on va effectuer, et donc de se servir de modèles simplifiés par rapport au cas général.

Si l'on applique cette démarche aux six cas présentés au début, il faut calculer par exemple les nombres $S_{T}, V_{R}, F_{D}$. $\mathscr{C}, \mathcal{M}$. Il s'agit bien sûr d'un ordre de grandeur de ces nombres, que l'on estime à partir des valeurs connues de certains paramètres (vitesse du son, viscosité,...) ou d'estimations pour d'autres (fréquence d'oscillation,....). Ces estimations devront bien sûr être confirmées a posteriori.

Dans la figure 3, on fait un premier tri de ces différents cas en fonction des nombres de Stokes et de la vitesse réduite. Alors que le cas $\mathrm{D}$ (peau de dauphin) nécessite à première vue de prendre en compte simultanément le mouvement permanent du fluide et les oscillations de la structure $\left(V_{R} \simeq 1\right)$, on peut dans les autres cas négliger l'un ou l'autre. De même, les valeurs de nombre de Stokes différencient clairement les cas A, B, D, E des cas C et F, qui sont les deux systèmes de petite taille, où les effets visqueux prennent une grande importance relative.

Les autres nombres (Froude, Compressibilité et Masse) (fig. 4) permettent également de repérer les effets dominants.

\subsection{Choix d'une modélisation}

Dans l'espace de dimension cinq $\left(S_{T}, V_{R}, F_{D}, \mathscr{C}, M\right)$ chaque exemple se situe dans une zone bien particulière. Les modélisations que l'on peut leur appliquer sont donc différentes

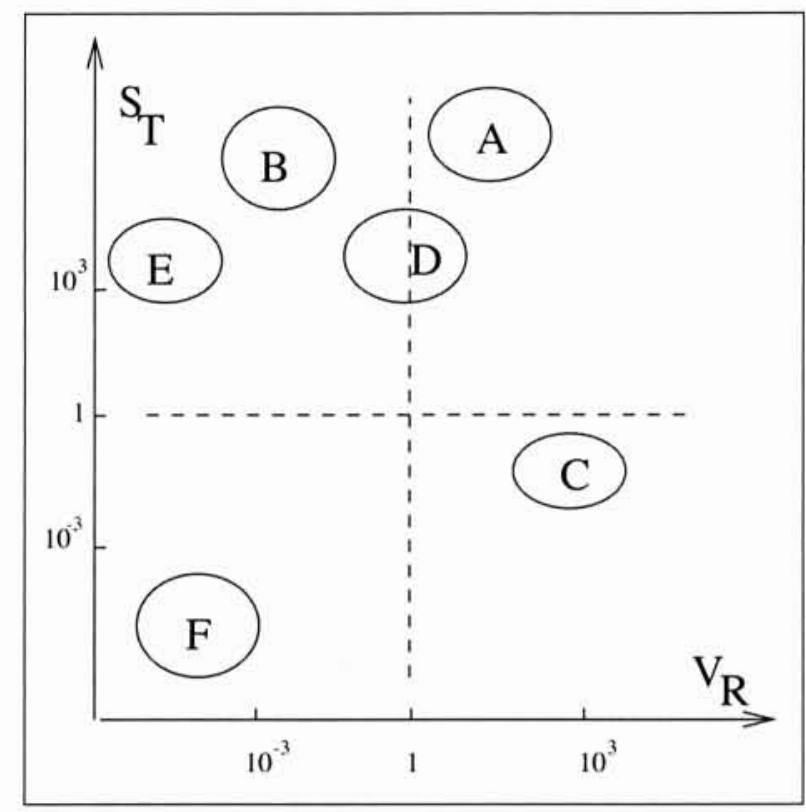

3. Vitesse réduite et nombre de Stokes.

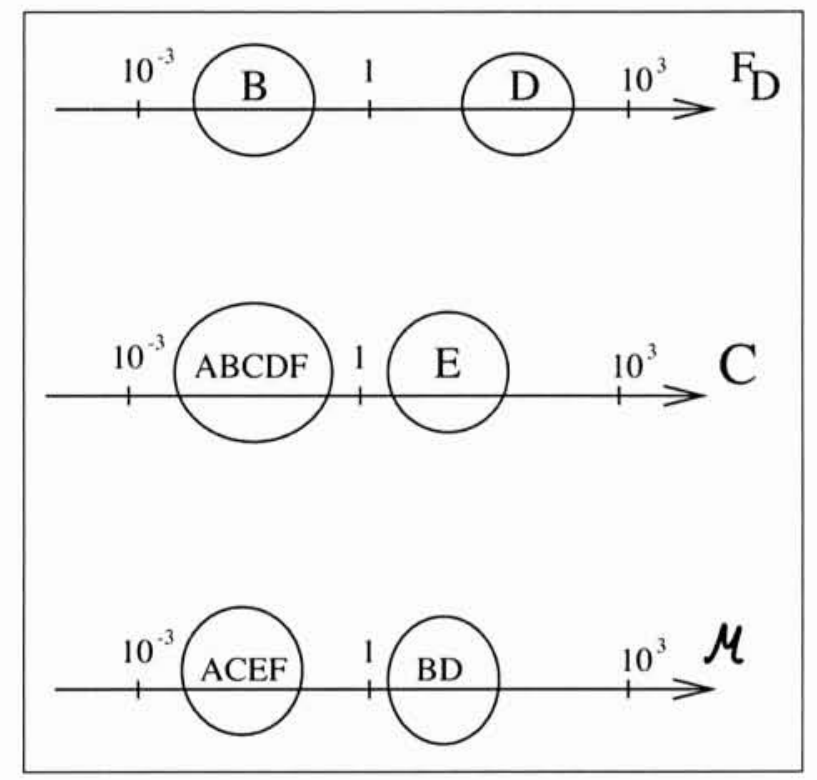

4. Nombres de Froude dynamique, de compressibilité et de masse.

mais beaucoup plus simples qu'une modélisation très générale de tous les effets.

Le cas A (flottement d'aileron F117) se rattache clairement au domaine $V_{R} \gg 1$ appelé couramment aéroélasticité, $[10,11]$. L'exemple B (barrage souple) sera traité à l'aide d'une modélisation avec fluide incompressible au repos et effet de surface libre [12]. L'exemple C (tête de le lecture) fait appel à des modèles moins classiques d'aéroélasticité avec effets visqueux dominants $\left(V_{R} \gg 1, S_{T}<1\right)$.

L'exemple D (traînée du dauphin) renvoie à ce qui est souvent appelé couplage fort [13]. Clairement, l'exemple E (rupture d'un verre) se rapporte à la vibro-acoustique [14].

Enfin, le cas $\mathrm{F}$ nécessite une modélisation en fluide visqueux incompressible, au repos. 


\section{IV $\square$ CONCLUSIONS}

Une des premières difficultés dans la modélisation des interactions fluide-structure est tout simplement de s'y retrouver dans la très grande variété des configurations. Pour cela, on recommande une approche par l'analyse dimensionnelle qui permet rapidement d'éliminer certains effets comme négligeables devant d'autres et donc de se raccrocher à des modélisations connues. Ces nombres adimensionnels permettent également d'utiliser un langage plus précis que les terminologies courantes.

\section{RÉFÉRENCES}

[1] M.L. WALD M.L. (1997). - Fluttery air deflector led to stealth crash. International Herald Tribune, september,17.

[2] Mysore G.V., Liapis S.I. and Plaut R.H. (1998). - Dynamic analysis of single-anchor inflatable dams. Journal of Sound and Vibration, 215(2): 251-272.

[3] WITELSKI T.P. (1998), - Dynamics of air bearing sliders. Physics of Fluids, 10(3) : 698-708.

[4] YEO K.S. (1998), - Optimization of viscoelastic compliant walls for transition delay. AIAA Journal, 36(4) : 656.
[5] Ho C.M., TAI Y.C. (1998). - Micro-electro-mechanicalsystems and fluid flows. Annual Review of Fluid Mechanics, $30:$ 579-612.

[6] Darrozes J.S., François C. (1982). - Mécanique des fluides incompressibles. Lecture Notes in Physics. SpingerVerlag, Berlin.

[7] BONNET A., LUNEAU J. (1989). - Théorie de la dynamique des fluides. Cepadues-Editions, Toulouse.

[8] CANDEl (S. (1995). - Mécanique des fluides. Dunod, Paris.

[9] Huerre P. (1998). - Mécanique des fluides. Ecole Polytechnique.

[10] FUNG Y.C. (1955). - An introduction to the theory of aeroelasticity. Dover, New-York.

[11] Dowell. H. (1995), - A modern course in aeroelasticity. Kluwer Academic Publishers, Dordrecht.

[12] GiBERT (R.J. (1988). - Vibrations des structures. Eyrolles.

[13] PaIDoussis M.P. (1988). - Fluid-structure Interactions. Slender Structures and Axial Flow. Volume 1. Academic Press.

[14] LesUeUR C. (1988). - Rayonnement acoustique des structures. Eyrolles. 